\title{
Prevalência da Deficiência e Associações Clínicas em Mulheres Sobreviventes ao Câncer de Mama: um Estudo-Piloto
}

doi: https://doi.org/10.32635/2176-9745.RBC.2020v66n2.843

\author{
Prevalence of Disability and Clinical Associations in Breast Cancer Women Survivors: a Pilot Study \\ Prevalencia de Discapacidad y Asociaciones Clínicas en Mujeres que Sobreviven al Cáncer de Mama: un Estudio Piloto
}

Adriano Lourenço'; Amanda Almeida Gomes Dantas²; Diego Neves Araújo³; Diego de Sousa Dantas

Resumo

Introduçáo: $\mathrm{O}$ tratamento do câncer de mama proporciona aumento da sobrevida e reduz a mortalidade, mas traz limitaçóes funcionais e efeitos colaterais que comprometem a sobrevida. Objetivo: Avaliar a prevalência da deficiência e sua associação com capacidade física, sono, fadiga e qualidade de vida em mulheres sobreviventes ao câncer de mama. Método: Trata-se de um estudo-piloto envolvendo 32 mulheres com, pelo menos, um ano após o tratamento clínico. Os dados da pesquisa foram obtidos por meio de questionários e um teste de caminhada de seis minutos. Resultados: A prevalência de deficiência na amostra foi $>90 \%$. O World Health Organization Disability Assessment Schedule 2.0 (WHODAS 2.0) revelou boa consistência interna ( $\alpha=0,874)$. A deficiência correlacionou-se forte e positivamente com cognição ( $r s=0,758)$, mobilidade ( $r s=0,709)$, atividade domésticas/trabalho ( $r s=0,718)$ e participação ( $r=0,701)$; e moderada e negativamente com fadiga $(\mathrm{r}=-0,621)$ e o Functional Assessment of Cancer Therapy-Fatigue (FACT-F) ( $\mathrm{r}=-0,672)$; e positivamente com o Disabilities of the Arm and Shoulder (DASH) ( $\mathrm{r}=0,639)$. Comparando-se os grupos de deficiência, observou-se diferença significativa com piores escores para o DASH $(\mathrm{d}=1,62)$, FACT-F $(\mathrm{d}=1,47)$, fadiga $(\mathrm{d}=1,21)$ e o indice de qualidade do sono de Pittsburgh (PSQI) (d=0,98). Conclusáo: Mulheres sobreviventes ao câncer de mama apresentaram algum nível de deficiência associada à fadiga, à pior qualidade do sono e à qualidade de vida, demonstrando impactos importantes na saúde dessas mulheres.

Palavras-chave: Neoplasias da Mama; Sobreviventes de Câncer; Estudos Transversais; Classificação Internacional de Funcionalidade, Incapacidade e Saúde; Perfil de Impacto da Doença.

\begin{abstract}
Introduction: The treatment of breast cancer improves survival and reduces mortality, but brings functional limitations and side effects that compromise survival. Objective: To evaluate the prevalence of disability and its association with physical capacity, sleep, fatigue and quality of life in women surviving breast cancer. Method: Pilo study involving 32 women at least one year after clinical treatment. The study data were obtained through questionnaires and a six-minute walk test. Results: The prevalence of disability in the sample was $>90 \%$. The World Health Organization Disability Assessment Schedule 2.0 (WHODAS 2.0) revealed good internal consistency $(\alpha=0.874)$. Disability correlated strongly and positively with cognition ( $\mathrm{rs}=0.758)$, mobility $(\mathrm{r} s=0.709)$, household/work activity $(\mathrm{r} s=0.718)$ and participation $(\mathrm{r}=0.701)$ and moderately and negatively with fatigue ( $\mathrm{r}=-0.621)$ and Functional Assessment of Cancer Therapy-Fatigue (FACT-F) ( $\mathrm{r}=-0.672)$ and positively with the Disabilities of the Arm and Shoulder (DASH) ( $r=0.639)$. Comparing the disability groups, a significant difference was observed with worse scores for DASH $(\mathrm{d}=1.62)$, FACT-F $(\mathrm{d}=1.47)$, fatigue $(\mathrm{d}=1.21)$ and the Pittsburgh Sleep Quality Index (PSQI) $(d=0.98)$. Conclusion: Women who survived breast cancer had some level of disability associated to fatigue, poor sleep quality and quality of life, demonstrating important impacts on the health of these women.

Key words: Breast Neoplasms; Cancer Survivors; Cross-Sectional Studies; International Classification of Functioning, Disability and Health; Sickness Impact Profile.
\end{abstract}

Resumen

Introducción: El tratamiento del cáncer de seno mejora la supervivencia y reduce la mortalidad, pero aún conlleva limitaciones funcionales y efectos secundarios que comprometen la supervivencia. Objetivo: Evaluar la prevalencia de discapacidad y su asociación con la capacidad física, el sueño, la fatiga y la calidad de vida en mujeres sobrevivientes de cáncer de seno. Método: Este es un estudio piloto con 32 mujeres al menos un año después del tratamiento clínico. Los datos de la encuesta se obtuvieron mediante cuestionarios y una prueba de caminata de seis minutos. Resultados: La prevalencia de discapacidad en la muestra fue $>90 \%$. El Programa de Evaluación de Discapacidad de la Organización Mundial de la Salud 2.0 (WHODAS 2.0) reveló una buena consistencia interna $(\alpha=0,874)$. La discapacidad se correlacionó fuertemente y positivamente con la cognición ( $r s=0,758)$, la movilidad ( $r s=0,709)$, la actividad del hogar/trabajo ( $r s=0,718)$ y la participación $(\mathrm{r}=0,701)$ y moderada y negativamente con la fatiga ( $\mathrm{r}=-0,621)$ y lo Functional Assessment of Cancer Therapy-Fatigue (FACT-F) ( $r=-0,672)$ y positivamente con lo Disabilities of the Arm and Shoulder (DASH) $(r=0,639)$. Comparando los grupos de discapacidad, se observó una diferencia significativa con peores puntajes para DASH $(d=1,62)$, FACT-F $(\mathrm{d}=1,47)$, fatiga $(\mathrm{d}=1,21)$ y el índice de calidad del sueño de Pittsburgh (PSQI) $(\mathrm{d}=0,98)$. Conclusión: Las mujeres que sobrevivieron al cáncer de seno tenían algún nivel de discapacidad asociada con la fatiga y la mala calidad del sueńo y la calidad de vida, lo que demuestra importantes impactos en la salud de estas mujeres.

Palabra clave: Neoplasias de la Mama; Supervivientes de Cáncer; Estudios Transversales; Clasificación Internacional del Funcionamiento, de la Discapacidad y de la Salud; Perfil de Impacto de Enfermedad.

\footnotetext{
${ }^{1}$ Universidade Federal do Rio Grande do Norte (UFRN). Programa de Pós-Graduação em Ciências da Reabilitação. Santa Cruz (RN), Brasil. Orcid iD: https://orcid. org/0000-0002-2463-0563

2 UFRN. Programa de Pós-Graduação em Saúde Coletiva. Santa Cruz (RN), Brasil. Orcid iD: https://orcid.org/0000-0002-7621-086X

${ }^{3}$ Centro de Ensino Superior e Desenvolvimento da Faculdade de Ciências Sociais Aplicadas (Cesed/Unifacisa). Campina Grande (PB), Brasil. Orcid iD: https://orcid. org/0000-0002-9541-5852

${ }^{4}$ UFRN. Programa de Pós-Graduação em Ciências da Reabilitação. Santa Cruz (RN), Brasil. Orcid iD: https://orcid.org/0000-0002-1966-3352

Endereço para correspondência: Diego de Sousa Dantas. UFRN. Faculdade de Ciências da Saúde do Trairi. Rua Theodorico Bezerra, 2 -122. Santa Cruz (RN), Brasil.
} CEP 59200-000. E-mail: diegodantas1@gmail.com 


\section{INTRODUÇÃO}

O tratamento do câncer de mama, apesar de proporcionar um aumento da taxa de sobrevida e reduçáo das tendências de mortalidade ${ }^{1,2}$, em razão dos avanços terapêuticos, com intervençóes eficientes e menos agressivas ${ }^{1,3}$, traz uma série de limitações funcionais, bem como o surgimento de efeitos colaterais crônicos, que ocasionam severo comprometimento ao longo da sobrevida livre da doençá.

Evidências atuais indicam que as causas que provocam limitaçóes funcionais, comportamentais e psicológicas durante toda a trajetória da vida de pacientes com câncer de mama são multifacetadas e estão associadas com prejuízos na qualidade de vida e com outras associaçóes clínicas que afetam a saúde dessas mulheres ${ }^{5,6}$.

Os prejuízos funcionais que limitam a execução de atividades e restringem a participação social dessas mulheres, de acordo com a Classificação Internacional de Funcionalidade, Incapacidade e Saúde $(\mathrm{CIF})^{7}$, podem ser entendidos como deficiências, representando aspectos negativos da interação entre as estruturas e funçóes biológicas, atividade e participação. $\mathrm{E} o$ entendimento dessa deficiência deve envolver a relação do indivíduo com os fatores contextuais (ambientais e pessoais) ${ }^{7}$.

Nessa perspectiva, é importante observar que o tratamento do câncer de mama ainda causa impactos negativos de curto a longo prazo na funcionalidade e, quando associados a outros problemas, podem desencadear seríssimos prejuízos à saúde ${ }^{5,8}$.

Estudos sobre a funcionalidade/deficiência apontam que o questionário World Health Organization Disability Assessment Schedule 2.0 (WHODAS 2.0) é um instrumento confiável e válido para ser usado em pacientes com câncer de mama, sendo utilizado em diferentes países no mundo, demonstrando-se útil para mensurar a prevalência de deficiência, avaliada pela limitação de atividades e restriçóes de participação ${ }^{9,10}$. Sendo assim, a utilização de um questionário padrão baseado na CIF pode gerar dados importantes e comparáveis com outras populaçóes para o desenvolvimento e o planejamento de condutas terapêuticas de acordo com a condição de saúde da paciente ${ }^{9,10}$. Porém, ainda há escassez de dados com mulheres sobreviventes ao câncer de mama e ao longo da sobrevida livre da doença.

Diante dessas evidências, surge a necessidade de dar atenção para as deficiências e as consequências associadas a estas, em mulheres sobreviventes ao câncer de mama. Pois, essas deficiências sinalizam comprometimentos na execução de atividades específicas e participação e, consequentemente, interferem negativamente no bem-estar biopsicossocial dessa população. A falta de conhecimento dessas manifestaçóes provocam dificuldades nas avaliaçóes e condutas terapêuticas direcionadas a essas mulheres ${ }^{6,11}$.

Desse modo, este estudo objetivou avaliar a prevalência da deficiência e sua associação com a capacidade física, fadiga, sono e qualidade de vida em mulheres sobreviventes ao câncer de mama.

\section{MÉTODO}

Trata-se de um estudo-piloto, realizado com mulheres sobreviventes ao câncer de mama, executado entre os meses de janeiro e setembro de 2018. Foi desenvolvido em acordo com a Declaração de Helsinki e aprovado pelo Comitê de Ética em Pesquisa local, sob o número CAAE: 67839617.9.0000.5568.

Foram incluídas no estudo mulheres com idade superior a 18 anos, diagnóstico de câncer de mama, estágios clínicos de I a III (tumor limitado à mama e aos nódulos linfáticos), que tenham finalizado o tratamento clínico há pelo menos um ano. Foram estabelecidos como critérios de exclusão: a) mulheres com câncer de mama bilateral; b) recidiva locorregional ou metástase; c) alteração neurológica ou cognitiva que impedisse a aplicação dos questionários e teste físico; ed) instabilidade hemodinâmica que contraindicasse a realização do teste físico ${ }^{12}$.

A seleção dessas pacientes foi realizada na comunidade junto aos serviços de saúde da atenção primária à saúde dos municípios de Santa Cruz e Currais Novos, ambos situados no Estado do Rio Grande do Norte, com população de 35.797 e 42.652 habitantes e índice de desenvolvimento humano (IDH) de 0,635 e 0,691, respectivamente ${ }^{13}$.

Todas as participantes do estudo foram esclarecidas em relação aos objetivos da pesquisa, benefícios da participação e anonimato e consentiram participar do estudo por meio da assinatura do Termo de Consentimento Livre e Esclarecido.

As participantes foram submetidas a uma avaliação que englobava dados gerais de identificação, história de saúde, questionários validados, medidas antropométricas e testes físicos. Todas as avaliaçóes foram executadas por dois avaliadores previamente treinados e calibrados para os instrumentos e testes aplicados.

\section{MEDIDAS UTILIZADAS}

As variáveis sociodemográficas incluíram: idade (entre 32-59 anos, $\geq 60$ anos), raça/etnia (branca, parda, negra, amarela), estado conjugal (sem companheiro, com companheiro), escolaridade (até 8 anos, entre 8-11 anos, $>11$ anos), ocupação antes e após tratamento e renda familiar (até 1 salário mínimo, >1 salário mínimo). No 
tocante às variáveis clínicas, foram investigados: índice de massa corporal (IMC) categorizado (normal, sobrepeso, obeso), estágio da doença (I, II ou III), tratamento adjuvante local, tratamento sistêmico em algum momento, tratamento adjuvante sistêmico, procedimento cirúrgico (mastectomia radical, quadrantectomia/ setorectomia), linfadenectomia, linfedema, histórico familiar de câncer, equipe multidisciplinar, diabetes mellitus, hipertensão arterial sistêmica (HAS), artritel artrose e outras comorbidades.

A aferição da estatura e do peso corporal das pacientes foi realizada com estadiômetro de marca Heigth e balança digital de marca Cadence, respectivamente. O IMC foi calculado por meio da razão entre o peso corporal e a estatura elevada ao quadrado (IMC $=$ peso/altura ${ }^{2}$ ).

As variáveis analisadas foram deficiência, incapacidade de membro superior homolateral ao câncer, fadiga, qualidade de vida, qualidade do sono e capacidade física.

O nível de deficiência foi avaliado por meio do WHODAS 2.0 de 36 questóes, validado para o português e aplicado mediante entrevista. Foi desenvolvido com base na $\mathrm{CIF}^{14}$. O instrumento estabelece o nível de deficiência por meio de seis domínios: cognição, mobilidade, autocuidado, relaçóes interpessoais, atividades de vida e participação. $\mathrm{O}$ escore total do instrumento varia de 0 (nenhuma deficiência) a 100 (deficiência completa) ${ }^{10,15}$.

A incapacidade de membros superiores foi mensurada por intermédio do questionário Disabilities of the Arm and Shoulder (DASH) com 30 questóes que avaliam atividades executadas na semana anterior, denominadas para medir o nível de deficiência nas funçôes físicas, sociais e ocupacionais e na presença de sintomas. $\mathrm{O}$ escore total da escala varia de 0 (sem incapacidade) a 100 (grave incapacidade) $)^{16,17}$.

A qualidade de vida e a fadiga das pacientes foram avaliadas por meio do questionário Functional Assessment of Cancer Therapy-Fatigue (FACT-F), constituído por 40 questóes com cinco domínios que pontuam separadamente: bem-estar físico (0-28 pontos); bem-estar social/familiar (0-28 pontos); bem-estar emocional (0-24 pontos); bem-estar funcional (0-28 pontos) e a subescala de fadiga (0-52 pontos). Cada item tem pontuação que varia de 0 a 4 . O escore total varia de 0 a 160 pontos, e os altos escores indicam melhor qualidade de vida e menor ocorrência de fadiga ${ }^{18,19}$.

A qualidade do sono foi avaliada por meio do indice de qualidade do sono de Pittsburgh (PSQI), composto por sete componentes que avaliam o sono separadamente em qualidade subjetiva, distúrbio, latência, duração, eficiência, uso de medicamentos e sonolência diurna e distúrbios durante o dia, com pontuação que varia de 0-3 pontos, e a soma dos componentes fornece um escore total de 21 pontos, no qual pontuaçóes mais baixas indicam melhor qualidade do sono ${ }^{20,21}$.

A capacidade física foi mensurada por meio do teste de caminhada de seis minutos (TC6), que consiste em um teste de capacidade submáxima, de baixo custo e fácil execução, seguro e reprodutível com validação para população de mulheres com câncer de mama ${ }^{22,23}$. $\mathrm{O}$ teste foi baseado nos preceitos da American Thoracic Society (ATS), no qual as participantes foram instruídas para caminhar em um corredor plano e firme de 30 metros durante seis minutos em velocidade máxima sustentada. Foram registradas, no início, durante e no fim do teste, a frequência cardíaca e a saturação periférica de oxigênio $\left(\mathrm{SpO}_{2}\right)$ por meio do oxímetro de pulso (modelo CMS50N - Contec, São Paulo, Brasil), assim como, durante o teste, foram registradas a percepção subjetiva de esforço para dispneia (Borg-D) e a fadiga de membros inferiores (Borg-F) por meio da escala de Borg modificada. A mensuração da distância prevista no TC6 foi pela equação desvio-padrão $\left(2,11_{*}\right.$ altura $\left.\mathrm{cm}\right)$ - (2,29* peso kg) - (5,78*idade $)+667 \mathrm{~m}$, e a capacidade funcional foi estimada a partir da relação da distância obtida no teste pela distância prevista ${ }^{24}$.

\section{ANÁLISES ESTATÍSTICAS}

Utilizou-se estatística descritiva e inferencial na análise de dados, empregando o software SPSS 20.0 (Statistical Package for Social Science). As variáveis categóricas foram mostradas em frequências absoluta e relativa e as variáveis contínuas foram expostas em média e desvio-padrão, mediana, intervalo interquartil. A normalidade dos dados contínuos foi feita pelo teste de Shapiro-Wilk.

Para analisar a consistência interna do instrumento WHODAS 2.O, foi utilizado o alpha de Cronbach $(\alpha)$, que pode variar de 0 a 1 , de forma que, quanto mais próximo de um, mais forte e consistente será a correlação entre seus itens. A correlação entre as variáveis quantitativas geradas pelo WHODAS 2.0, DASH e FACT-F foi calculada usando o coeficiente de correlação de Spearman e/ou Pearson.

Para análises comparativas, as mulheres foram divididas em dois grupos: deficiência leve e deficiência moderada. Os testes t de Student e de Mann-Whitney foram empregados para comparação de médias e medianas entre grupos quando os dados foram paramétricos e não paramétricos, respectivamente. $\mathrm{O}$ intervalo de confiança 95\% para a diferença entre os grupos foi calculado.

A significância clínica dos resultados foi analisada por meio do teste d de Cohen. O tamanho do efeito foi interpretado da seguinte maneira, valores $\geq 0,2$ foram classificados como baixo efeito clínico; $\geq 0,5$ moderado; e $\geq 0,8$ alto $^{25}$. O nível de significância de $\mathrm{p}<0,05$ foi adotado para todos os testes. 


\section{RESULTADOS}

Inicialmente, foram identificadas 101 mulheres com diagnósticos de câncer. Destas, 36 não eram elegíveis, pois tinham outros tipos de câncer (ovário; pele; cabeça e pescoço). Das 65 mulheres elegíveis, foram excluídas 28 mulheres, cinco recusaram-se a participar da pesquisa e a amostra final foi composta por 32 mulheres. A caracterização social e clínica da amostra está apresentada nas Tabelas 1 e 2, respectivamente.

A maioria das mulheres incluídas estava na faixa etária de 32-59 anos (78\%), se declarava de raça parda (50\%) e convivia com companheiro (53\%). Com relação ao perfil clínico, as participantes foram diagnosticadas em sua maioria nos estágios II e III (62\%), estavam com excesso de peso no momento da avaliação (44\%), e possuíam histórico familiar de câncer $(75 \%)$. O principal procedimento cirúrgico realizado foi mastectomia radical $(53 \%)$ e $81 \%$ das participantes foram submetidas ao esvaziamento axilar associado à cirurgia.

Das 32 mulheres avaliadas, 21 apresentaram deficiência moderada $(72 \%)$, oito deficiência leve $(25 \%)$, uma possuía deficiência severa (3\%) e duas não apresentavam deficiência $(6,25 \%)$.

Tabela 1. Dados sociodemográficos das mulheres sobreviventes ao câncer de mama no período de janeiro a setembro de 2018

\begin{tabular}{lcc}
\hline \multicolumn{1}{c}{ Variáveis } & n & $\%$ \\
\hline Idade & 25 & 78,1 \\
$32-59$ anos & 7 & 21,9 \\
$\geq 60$ anos & & \\
Raça/etnia & 10 & 31,3 \\
Branca & 16 & 50,0 \\
Parda & 2 & 6,3 \\
Negra & 4 & 12,5 \\
Amarela & & \\
Estado conjugal & 15 & 46,9 \\
Sem companheiro & 17 & 53,1 \\
Com companheiro & & \\
Escolaridade & 9 & 28,4 \\
Até 8 anos & 13 & 40,3 \\
Entre 8-11 anos & 10 & 31,3 \\
>11 anos & 24 & 75,0 \\
Ocupaçáo antes do tratamento & 9 & 28,1 \\
Ocupaçáo após tratamento & & \\
Renda familiar & 12 & 37,5 \\
até 1 salário mínimo & 20 & 62,5 \\
\hline >1 salário mínimo & & \\
\hline
\end{tabular}

Tabela 2. Caraterização clínica das mulheres sobreviventes ao câncer de mama no período de janeiro a setembro de 2018

\begin{tabular}{|c|c|c|}
\hline Variáveis & $\mathbf{n}$ & $\%$ \\
\hline \multicolumn{3}{|l|}{ IMC categorizado } \\
\hline Peso normal & 6 & 18,8 \\
\hline Sobrepeso & 14 & 43,8 \\
\hline Obeso & 12 & 37,5 \\
\hline \multicolumn{3}{|l|}{ Estágio da doença } \\
\hline Estágio I & 12 & 37,5 \\
\hline Estágios II ou III & 20 & 62,5 \\
\hline \multicolumn{3}{|l|}{ Tratamento local } \\
\hline Radioterapia adjuvante & 29 & 90,6 \\
\hline \multicolumn{3}{|l|}{ Tratamento sistêmico } \\
\hline Quimioterapia em algum momento & 30 & 93,8 \\
\hline Hormonioterapia adjuvante & 12 & 37,5 \\
\hline \multicolumn{3}{|l|}{ Procedimento cirúrgico } \\
\hline Mastectomia radical & 17 & 53,1 \\
\hline Quadrantectomia/setorectomia & 15 & 46,9 \\
\hline Linfadenectomia & 26 & 81,3 \\
\hline Linfedema & 18 & 56,3 \\
\hline Histórico familiar de câncer & 24 & 75,0 \\
\hline Equipe multidisciplinar & 24 & 75,0 \\
\hline Diabetes mellitus & 6 & 18,8 \\
\hline Hipertensão arterial sistêmica & 10 & 31,3 \\
\hline Artrite/artrose & 3 & 9,4 \\
\hline Outras comorbidades & 18 & 56,3 \\
\hline
\end{tabular}

O WHODAS 2.0 de 36 questôes demonstrou boa consistência interna para pacientes com câncer de mama $(\alpha=0,874)$. Os escores do WHODAS, total e por domínios, bem como os coeficientes de correlação entre os domínios do questionário com o escore total, são apresentados na Tabela 3.

Quando comparado a outros questionários, observou-se correlação moderada e negativa entre o escore total do WHODAS e o escore total do FACT-F $(r=-0,672)$ e os domínios subescala de fadiga $(\mathrm{r}=-0,621)$ e bem-estar físico ( $\mathrm{rs}=-0,684)$. Os domínios bem-estar emocional $(\mathrm{r}=-0,374)$ e bem-estar funcional $(r=-0,372)$ apresentaram correlação significativa, fraca e negativa. O domínio bem-estar social/ familiar não revelou significância estatística. A correlação entre o escore total do WHODAS e o escore total do DASH foi de $r=0,639$, indicando correlação moderada e positiva.

A fim de avaliar a associação da deficiência no desempenho em testes específicos e questionários validados, a amostra de mulheres com deficiência foi dividida em dois grupos: deficiência leve e moderada, 
Tabela 3. Caracterização da amostra de acordo com o WHODAS $2.0(n=32)$

\begin{tabular}{lccccc}
\hline WHODAS 2.0 & $\begin{array}{c}\text { Valores } \\
\text { mínimo- } \\
\text {-máximo }\end{array}$ & $\begin{array}{c}\text { Média } \\
\text { desvio- } \\
\text {-padrão }\end{array}$ & $\begin{array}{c}\text { Mediana } \\
\text { (intervalo } \\
\text { interquartil) }\end{array}$ & $\begin{array}{c}\text { Correlação } \\
\text { com o }\end{array}$ & $\begin{array}{c}\text { escore total } \\
\text { palor }\end{array}$ \\
\hline Escore total & $0-58,7$ & $27,07 \pm 13,83$ & 28,80 & 1.00 & - \\
Cognição & $0-60,0$ & $23,28 \pm 18,08$ & $20,00(32,50)$ & rs $=0,758$ & $<0,001$ \\
Mobilidade & $0-81,2$ & $25,98 \pm 22,46$ & $18,75(37,50)$ & rs=0,709 & $<0,001$ \\
Autocuidado & $0-50,0$ & $11,25 \pm 14,53$ & $10,00(20,00)$ & rs $=0,558$ & 0,001 \\
Atividades de vida diária & $0-100,0$ & $46,56 \pm 34,70$ & $40,00(60,00)$ & rs $=0,718$ & $<0,001$ \\
Relações interpessoais & $0-75,0$ & $19,01 \pm 18,35$ & $16,67(14,59)$ & rs $=0,555$ & 0,001 \\
Participação & $0-83,3$ & $33,46 \pm 19,50$ & $33,33(29,16)$ & $r=0,701$ & $<0,001$ \\
\hline
\end{tabular}

Legendas: $r=$ Correlação de Pearson; rs=Correlação de Spearman.

Nota: $\mathrm{O}$ valor de p refere-se aos testes de correlação. O escore total e por domínios varia de 0 a 100.

de acordo com o escore total do WHODAS 2.0. Dessa análise estatística, exclui-se o caso de deficiência severa. As comparaçôes são apresentadas na Tabela 4.

Observa-se, na Tabela 4, diferença significativa com piores escores totais para todas as escalas aplicadas $(\mathrm{p}<0,05)$. Apenas o domínio bem-estar social/familiar, assim como os componentes qualidade subjetiva, distúrbios, latência, duração e eficiência do sono não apresentaram diferença significativa nos escores entre os grupos. Por meio do d de Cohen, foi possível comprovar o forte efeito negativo da deficiência no desempenho funcional do membro superior $(\mathrm{d}=1,62)$, qualidade de vida $(\mathrm{d}=1,47)$, fadiga $(\mathrm{d}=1,21)$ e qualidade do sono $(\mathrm{d}=0,98)$. Assim como nos domínios bem-estar físico $(\mathrm{d}=1,54)$, emocional $(\mathrm{d}=0,98)$ e funcional ( $d=1,07)$, e no componente distúrbio do sono durante o dia $(\mathrm{d}=1,11)$. A comparaçẫo das variáveis avaliadas por meio do TC6 não apresentou diferença significativa entre os grupos.

\section{DISCUSSÃO}

O presente artigo verificou que $94 \%$ das mulheres apresentam algum grau de deficiência no período de sobrevida livre da doença, com maiores escores de dificuldade para as atividades de vida e participação. $\mathrm{O}$ escore do WHODAS esteve associado a menor bem-estar físico e desempenho funcional do membro superior ipsilateral, maior intensidade de fadiga e baixa qualidade de vida geral.

Embora exista uma lacuna de conhecimento acerca de estudos que avaliem a prevalência e efeitos da deficiência em pacientes sobreviventes ao câncer com o WHODAS 2.0, estudos prévios, com outros instrumentos de medida, reportam que a dificuldade na execução de atividades do cotidiano impacta negativamente no bem-estar dessas mulheres $3,6,11,26-28$.
Estudos apontam que mulheres sobreviventes ao câncer de mama tendem a experenciar a curto ou longo prazo prejuízos na saúde física e cognitiva, no bem-estar psicológico e nas relações interpessoais, repercutindo em prejuízos na qualidade de vida ${ }^{5}$. Tais prejuízos decorrem em parte dos efeitos tardios dos tratamentos clínico e cirúrgico ${ }^{6,29}$.

Entre os prejuízos funcionais relacionados à cirurgia, a perda de desempenho funcional do membro superior é o principal. E estes podem desencadear a curto e a longo prazo outros déficits funcionais como dor, linfedema, limitação de movimentos articulares, parestesia e fraqueza muscular, impossibilitando que essas mulheres executem de forma satisfatória suas atividades habituais ${ }^{3,6,16,17}$.

Das repercussóes negativas provenientes do tratamento clínico do câncer de mama, a fadiga é um dos sintomas mais reportados entre as mulheres e caracteriza-se como um sintoma crônico e extenuante, que provoca cansaço intenso e baixo desempenho funcional em virtude da falta de disposição ${ }^{27}$. A existência de problemas do sono e fadiga tende ao agravamento dos prejuízos funcionais relatados 5 . E esses prejuízos podem se cronificar no tempo, pois, de acordo com Devoogdt et al..$^{30}$, mulheres após os tratamentos cirúrgico e clínico e ao longo da sobrevida não apresentaram recuperação satisfatória na capacidade física e na funcionalidade.

Com relação às alteraçôes do sono, os resultados referentes ao maior nível de deficiência estáo de acordo com Schmidt et al.$^{28}$, que observaram que maiores prejuízos na cognição, nas relações interpessoais e comportamentais, nas atividades domésticas/trabalho e participação social, domínios que compóem o WHODAS 2.0, desencadeiam a precariedade do sono. Os resultados aqui apresentados corroboram outros estudos, demonstrando que a precariedade do sono está associada à deficiência de mulheres sobreviventes ao câncer de mama ${ }^{31,32}$. 
Tabela 4. Comparação dos escores obtidos nos questionários e TC6 entre mulheres com deficiência leve e moderada

\begin{tabular}{|c|c|c|c|c|c|c|}
\hline Variável & $\begin{array}{l}\text { Amostra total } \\
\qquad(n=29)\end{array}$ & $\begin{array}{l}\text { Deficiência leve } \\
\qquad(n=8)\end{array}$ & $\begin{array}{c}\text { Deficiência } \\
\text { moderada } \\
(n=21)\end{array}$ & CI 95\% & Cohen's d & $p$ \\
\hline \multicolumn{7}{|l|}{ TC6 } \\
\hline Distância prevista² & $545,01 \pm 61,82$ & $550,75 \pm 44,40$ & $534,50 \pm 78,16$ & $-44,25-76,75$ & 0,26 & 0,586 \\
\hline Distância obtida' & $436,50(66,75)$ & $450,75(46,25)$ & $410,38(134,00)$ & $-20,00-99,00$ & 0,64 & 0,354 \\
\hline Número de passos² & $855,90 \pm 236,65$ & $985,25 \pm 158,75$ & $793,90 \pm 257,98$ & $-10,09-392,78$ & 0,89 & 0,062 \\
\hline Capacidade funcional $(\%)^{2}$ & $78,94 \pm 10,19$ & $82,52 \pm 10,09$ & $76,55 \pm 11,07$ & $-3,26-15,19$ & 0,56 & 0,196 \\
\hline DASH escore total ${ }^{2}$ & $33,02 \pm 17,46$ & $16,56 \pm 12,41$ & $39,01 \pm 15,19$ & $-34,83-(-10,07)$ & 1,62 & $0,001^{*}$ \\
\hline \multicolumn{7}{|l|}{ FACT-F } \\
\hline Escore total ${ }^{2}$ & $111,24 \pm 20,28$ & $129,15 \pm 11,85$ & $106,23 \pm 18,57$ & $8,35-37,48$ & 1,47 & $0,003^{*}$ \\
\hline Bem-estar físico $^{1}$ & $18,00(7,00)$ & $22,00(3,25)$ & $16,00(7,00)$ & $2,00-8,00$ & 1,54 & $0,002^{*}$ \\
\hline Bem-estar social/familiar ${ }^{1}$ & $21,00(7,68)$ & $21,50(1,88)$ & $21,00(8,59)$ & $-1,83-6,00$ & 0,52 & 0,432 \\
\hline Bem-estar emocional' ${ }^{1}$ & $22,00(5,00)$ & $22,00(1,00)$ & $19,00(5,50)$ & $0,00-5,00$ & 0,98 & $0,048^{*}$ \\
\hline Bem-estar funcional $\left.\right|^{2}$ & $18,00 \pm 4,47$ & $20,88 \pm 3,72$ & $16,71 \pm 4,06$ & $0,77-7,55$ & 1,07 & $0,018^{*}$ \\
\hline Subescala fadiga ${ }^{2}$ & $36,79 \pm 9,24$ & $43,63 \pm 5,29$ & $35,52 \pm 7,87$ & $1,89-14,32$ & 1,21 & $0,013^{*}$ \\
\hline \multicolumn{7}{|l|}{ PSQI } \\
\hline Escore total $\left.\right|^{2}$ & $7,72 \pm 3,22$ & $5,50 \pm 1,93$ & $8,33 \pm 3,60$ & $-5,60-(-0,07)$ & 0,98 & $0,045^{*}$ \\
\hline Qualidade subjetiva do sono ${ }^{\top}$ & $1,00(1,00)$ & $1,00(0,75)$ & $1,00(1,00)$ & $-1,00-0,00$ & 0,88 & 0,055 \\
\hline Distúrbios do sono ${ }^{1}$ & $2,00(1,00)$ & $1,00(1,00)$ & $2,00(1,00)$ & $-1,00-0,00$ & 0,45 & 0,261 \\
\hline Latência do sono ${ }^{1}$ & $2,00(1,75)$ & $2,00(1,75)$ & $2,00(1,50)$ & $0,00-1,00$ & 0,31 & 0,516 \\
\hline Duração do sono ${ }^{1}$ & $0,00(2,00)$ & $0,00(1,75)$ & $0,00(2,00)$ & $-1,00-0,00$ & 0,33 & 0,527 \\
\hline Eficiência do sono ${ }^{1}$ & $0,00(0,00)$ & $0,00(0,75)$ & $0,00(0,00)$ & $0,00-0,00$ & 0,27 & 0,502 \\
\hline $\begin{array}{l}\text { Uso de medicamento e sonolência } \\
\text { diurna' }\end{array}$ & $0,00(2,75)$ & $0,00(0,00)$ & $0,00(3,00)$ & $-3,00-0,00$ & 0,00 & $0,043^{*}$ \\
\hline Distúrbios durante o dia ${ }^{1}$ & $1,00(1,00)$ & $0,00(0,75)$ & $1,00(1,50)$ & $-1,00-0,00$ & 1,11 & $0,020^{*}$ \\
\hline
\end{tabular}

Legendas: ${ }^{1}$ Variável com distribuição não paramétrica. Dados expressos em mediana e intervalo interquartil. Utilizou-se o teste de Mann-Whitney para comparaçâo de mediana entre os grupos; ${ }^{2}$ Variável com distribuição paramétrica. Dados expressos em média e desvio-padrão. O teste t de Student realizou a comparação de medias. ${ }^{*} \mathrm{p}$ valor $<0,05$. DASH=Disabilities of the Arm and Shoulder; FACT-F=Functional Assessment of Cancer Therapy-Fatigue; PSQI= Pittsburgh Sleep Quality Index; IC 95\%=Intervalo de confiança e p valor.

Nota: Valores maiores de FACT-F representam melhor bem-estar, enquanto maiores escores de DASH e PSQI representam desfecho negativo.

Em relação à qualidade de vida, observou-se que as mulheres com maior nível de deficiência apresentam baixos escores da qualidade de vida (FACT-F), destacando-se piores escores para o bem-estar físico, bem-estar funcional, bem-estar emocional e fadiga, estando congruentes com os resultados de outros estudos ${ }^{28,33,34}$. Contudo, cabe ressaltar que neste estudo não se observou significância estatística entre piores níveis de deficiência e bem-estar social, avaliados pelo FACT-F, talvez esse acontecimento seja explicado pelo fato de $78,1 \%$ das participantes do estudo integrarem um grupo de apoio a mulheres com câncer, revelando a grande relevância do apoio social para essas mulheres.

Ao analisar os valores encontrados no TC6, observou-se que maiores níveis de deficiência não causaram alteraçôes hemodinâmicas. Embora a amostra tenha demonstrado um percentual aceitável de capacidade física (superior a 70\%), uma parcela significativa das mulheres foi categorizada com sobrepeso $(43,8 \%)$ e obesidade $(37,5 \%)$, sendo comum o ganho de peso durante e/ou após o tratamento do câncer ${ }^{35}$, que se caracteriza por um baixo nível de atividade física. Evidências científicas 
demostraram que o tratamento do câncer e as suas deficiências a longo prazo levam a baixos níveis de atividade física e capacidade física ${ }^{6,36}$.

Contudo, neste estudo, não se observou significância estatística entre nível de deficiência e capacidade física e isso pode ser explicado em virtude de o teste exigir capacidade submáxima dos membros inferiores e condicionamento cardiorrespiratório. Também se observou correlação entre piores escores de deficiência e mobilidade, divergindo dos resultados obtidos no TC6. Talvez essa divergência possa ser explicada pelo fato de o WHODAS 2.0 incluir no domínio mobilidade questôes que exijam maior capacidade física em relação ao TC6, tais como ficar em pé por mais de 30 minutos, sair de casa e enfrentar obstáculos no percurso e andar longas distâncias.

Diante da repercussão significativa do tratamento oncológico a longo prazo na vida dessas mulheres, destacam-se a importância e a necessidade de integrar informaçóes relacionadas a sintomas decorrentes dos tratamentos realizados, bem como sobre a deficiência nas avaliaçôes clínicas e nas condutas terapêuticas conduzidas com esse público. Esse registro de informaçóes voltadas à funcionalidade e à deficiência deve ser realizado em uma linguagem padronizada por meio de medidas válidas, baseadas na CIF e amplamente utilizadas, como o WHODAS, a fim de gerar dados relevantes, reprodutíveis e comparáveis com pacientes de diferentes localidades e contextos clínicos ${ }^{37}$.

Como limitaçóes deste trabalho, destaca-se o desenho transversal, que restringe a interpretação dos dados e associaçóes, passíveis de causalidade reversa, e impossibilita a inferência de causalidade. Além disso, observa-se que, em virtude de ser um estudo-piloto, o tamanho amostral pequeno impossibilitou a realizaçáo de modelos mais robustos, como regressōes multivariadas com a inclusão de variáveis de ajustes para as associaçóes verificadas. $\mathrm{O}$ pequeno tamanho amostral ressalta a dificuldade de identificaçáo e acesso a essas mulheres, nos territórios de saúde, após a conclusão do tratamento oncológico.

\section{CONCLUSÃO}

A partir deste estudo-piloto, pode-se concluir que mulheres sobreviventes ao câncer de mama, mesmo após o término do tratamento clínico, apresentaram algum nível de deficiência. Também foi identificado que a deficiência está associada a piores escores para o desempenho dos membros superiores ipsilateral à cirurgia, maior fadiga, pior qualidade do sono e qualidade de vida, demonstrando impactos importantes na saúde dessas mulheres.

Este estudo contribuiu para revelar problemas secundários que podem agravar o nível de deficiência e demonstrar a relevância e a necessidade de integrar informaçóes relacionadas sobre repercussôes negativas associadas à funcionalidade nas avaliaçóes clínicas e condutas terapêuticas direcionadas para as mulheres sobreviventes ao câncer de mama.

\section{CONTRIBUIÇÕES}

Adriano Lourenço contribuiu na coleta, elaboração do banco de dados e revisão crítica. Amanda Almeida Gomes Dantas colaborou na coleta de dados. Diego Neves Araújo colaborou na revisão crítica do manuscrito. Diego de Sousa Dantas contribuiu na concepção e planejamento do projeto de pesquisa; na análise e interpretaçáo dos dados; e na revisão crítica. Todos os autores aprovaram a versão final a ser publicada.

\section{DECLARAÇÃO DE CONFLITO DE INTERESSES}

Nada a declarar.

\section{FONTES DE FINANCIAMENTO}

Não há.

\section{REFERÊNCIAS}

1. Tao ZQ, Shi A, Lu C, et al. Breast cancer: epidemiology and etiology. Cell Biochem Biophys. 2015;72(2):333-8. doi: https://doi.org/10.1007/s12013-014-0459-6

2. Mathew A, George PS, Arjunan A, et al. Temporal trends and future prediction of breast cancer incidence across age groups in Trivandrum, South India. Asian Pacific J Cancer Prev. 2016;17(6):2895-9. doi: https://doi. org/10.1007/s002460010268

3. Carvalho FN, Bergmann A, Koifman RJ. Functionality in women with breast cancer: the use of International Classification of Functioning, Disability and Health (ICF) in clinical practice. J Phys Ther Sci. 2014;26(5):721-30. doi: https://doi.org/10.1589/jpts.26.721

4. Demiralp M, Oflaz F, Komurcu S. Effects of relaxation training on sleep quality and fatigue in patients with breast cancer undergoing adjuvant chemotherapy. J Clin Nurs. 2010;19(7-8):1073-83. doi: https://doi. org/10.1111/j.1365-2702.2009.03037.x

5. Peerawong T, Phenwan T, Supanitwatthana S, et al. Breast conserving therapy and quality of life in thai females: a mixed methods study. Asian Pacific J Cancer Prev. 2016;17(6):2917-21. doi: https://doi.org/10.1007/ s00520-016-3209-z

6. Oliveira NPD, Guedes TSR, Holanda AM, et al. Functional disability in women submitted to breast cancer treatment. Asian Pacific J Cancer Prev. 
2017;18(5):1207-14. doi: https://doi.org/10.22034/ APJCP.2017.18.5.1207

7. World Health Organization. How to use the ICF: a practical manual for using the International Classification of Functioning, Disability and Health (ICF) [Internet]. Geneva: WHO; 2013 Oct [cited 2019 June 12]. Available from: https://www.who.int/classifications/ drafticfpracticalmanual2.pdf?ua $=1$

8. Khan F, Amatya B, Pallant JF, et al. Factors associated with long-term functional outcomes and psychological sequelae in women after breast cancer. Breast. 2012;21(3):314-20. doi: https://doi.org/10.1016/j. breast.2012.01.013

9. Zhao HP, Liu Y, Li HL, et al. Activity limitation and participation restrictions of breast cancer patients receiving chemotherapy: psychometric properties and validation of the Chinese version of the WHODAS 2.0. Qual Life Res. 2013;22(4):897-906. doi: https://doi. org/10.1007/s11136-012-0212-9

10. Lee HH, Shin EK, Shin HI, et al. Is WHODAS 2.0 useful for colorectal cancer survivors?. Ann Rehabil Med. 2017;41(4):667-76. doi: https://doi.org/10.5535/ arm.2017.41.4.667

11. Assis MR, Marx AG, Magna LA, et al. Late morbidity in upper limb function and quality of life in women after breast cancer surgery. Brazilian J Phys Ther. 2013;17(3):236-43. doi: https://doi.org/10.1590/ S1413-35552012005000088

12. Herdy AH, Ritt LEF, Stein R, et al. Cardiopulmonary exercise test: background, applicability and interpretation. Arq Bras Cardiol. 2016;107(5):467-81. doi: https://doi. org/10.5935/abc. 20160171

13. Cidades@ [Internet]. Versão 4.4.4. Rio de Janeiro: IBGE. C2017 - [acesso 2019 jun 12]. Disponível em: https:// cidades.ibge.gov.br/

14. Ustun TB, Kostanjesek N, Chatterji S, et al., editors. Measuring Health and Disability: Manual for WHO Disability Assessment Schedule (WHODAS 2.0). Geneva: WHO; 2010.

15. Lee YH, Chang KH, Escorpizo R, et al. Accuracy of the World Health Organization Disability Assessment Schedule 2.0 (WHODAS 2.0) score as an objective assessment tool for predicting return-to-work status after head and neck cancer in male survivors. Support Care Cancer. 2019;27(2):433-441. doi: https://doi. org/10.1007/s00520-018-4322-y

16. Gummesson C, Atroshi I, Ekdahl C. The disabilities of the arm, shoulder and hand (DASH) outcome questionnaire: longitudinal construct validity and measuring self-rated health change after surgery. BMC Musculoskelet Disord. 2003;4:11. doi: https://doi. org/10.1186/1471-2474-4-11

17. Cheng HMS, Sampaio RF, Mancini MC, et al. Disabilities of the arm, shoulder and hand (DASH): factor analysis of the version adapted to Portuguese/ Brazil. Disabil Rehabil. 2008;30(25):1901-9. doi: https://doi.org/10.1080/09638280701749342

18. Ishikawa NM, Thuler LCS, Giglio, AG, et al. Reproducibility of Functional Assessment of Cancer Therapy-Fatigue (FACT-F) questionnaire for cancer patients. Appl Cancer Res [Internet]. 2008 [cited 2019 June 12];28(2):55-61. Available from: https://www. researchgate.net/publication/266160631

19. Ishikawa NM, Thuler LCS, Giglio AG, et al. Validation of the Portuguese version of Functional Assessment of Cancer Therapy-Fatigue (FACT-F) in Brazilian cancer patients. Support Care Cancer. 2010;18(4):481-90. doi: https://doi.org/10.1007/s00520-009-0697-0

20. Bertolazi AN, Fagondes SC, Hoff LS, et al. Validation of the Brazilian portuguese version of the pittsburgh sleep quality index. Sleep Med. 2011;12(1):70-5. doi: https:// doi.org/10.1016/j.sleep.2010.04.020

21. Otte JL, Davis L, Carpenter JS, et al. Sleep disorders in breast cancer survivors. Support Care Cancer. 2016;24(10):4197-205. doi: https://doi.org/10.1007/ s00520-016-3247-6

22. Brdareski Z, Djurović A, Susnjar S, et al. Physical activity and maximal aerobic capacity in breast cancer survivors: why this is important. Vojnosanit Pregl. 2014;71(1):6672. doi: https://doi.org/10.2298/VSP1401066B

23. Galiano-Castillo N, Arroyo-Morales M, Ariza-Garcia A, et al. The six-minute walk test as a measure of health in breast cancer patients. J Aging Phys Act. 2016 Oct;24(4):508515. doi: https://doi.org/10.1123/japa.2015-0056

24. Dourado VZ, Vidotto MC, Guerra RLF. Equaçôes de referência para os testes de caminhada de campo em adultos saudáveis. J Bras Pneumol. 2011;37(5):607-14. doi: https://doi.org/10.1590/S1806-37132011000500007

25. Héroux M. Cohen's d: how to interpret it?. 2017 July 27 [cited 2019 June 12]. In: Blog Scientifically Sound [Internet]. [place unknown: publisher unknown]. [2016]. https://scientificallysound.org/2017/07/27/ cohens-d-how-interpretation/

26. Koch L, Jansen L, Herrmann A, et al. Quality of life in long-term breast cancer survivors- A 10-year longitudinal population-based study. Acta Oncol. 2013;52(6):1119-28. doi: https://doi.org/10.3109/028 4186X.2013.774461

27. Ancoli-Israel S, Liu L, Rissling M, et al. Sleep, fatigue, depression, and circadian activity rhythms in women with breast cancer before and after treatment: A 1-year longitudinal study. Support Care Cancer. 2014;22(9):2535-45. doi: https://doi.org/10.1007/ s00520-014-2204-5

28. Schmidt ME, Wiskemann J, Steindorf K. Quality of life, problems, and needs of disease-free breast cancer survivors 5 years after diagnosis. Qual Life Res. 2018;27(8):207786. doi: https://doi.org/10.1007/s11136-018-1866-8 
29. Bellury L, Ellington L, Beck SL, et al. Older breast cancer survivors: can interaction analyses identify vulnerable subgroups? a report from the American Cancer Society studies of cancer survivors. Oncol Nurs Forum. 2013;40(4):325-36. doi: https://doi.org/10.1188/13. onf.325-336

30. Devoogdt N, van Kampen M, Geraerts I, et al. Physical activity levels after treatment for breast cancer: one-year follow-up. Breast Cancer Res Treat. 2010;123(2):417-25. doi: https://doi.org/10.1007/s10549-010-0997-6

31. Trudel-Fitzgerald C, Zhou ES, Poole EM, et al. Sleep and survival among women with breast cancer: 30 years of follow-up within the Nurses' Health Study. Br J Cancer. 2017;116(9):1239-46. doi: https://doi.org/10.1038/ bjc. 2017.85

32. Gonzalez BD, Lu Q. Sleep disturbance among Chinese breast cancer survivors living in the USA. Support Care Cancer. 2018;26(6):1695-8. doi: https://doi. org/10.1007/s00520-018-4128-y

33. Arndt V, Koch-Gallenkamp L, Jansen L, et al. Quality of life in long-term and very long-term cancer survivors versus population controls in Germany. Acta Oncol. 2017;56(2):190-7. doi: https://doi.org/10.1080/02841 86X.2016.1266089

34. Fontes F, Pereira S, Costa AR, et al. The impact of breast cancer treatments on sleep quality 1 year after cancer diagnosis. Support Care Cancer. 2017;25(11):3529-36. doi: https://doi.org/10.1007/s00520-017-3777-6

35. Doyle C, Kushi LH, Byers T, et al. Nutrition and physical activity during and after cancer treatment: an American Cancer Society guide for informed choices. CA Cancer J Clin. 2006;56(6):323-53. doi: https://doi.org/10.3322/ canjclin.56.6.323

36. Rogers LQ, Hopkins-Price P, Vicari S, et al. Physical activity and health outcomes three months after completing a physical activity behavior change intervention: persistent and delayed effects. Cancer Epidemiol Biomarkers Prev. 2009;18(5):1410-8. doi: https://doi.org/10.1158/1055-9965.EPI-08-1045

37. Dantas DS, Correa AP, Buchalla CM, et al. Biopsychosocial model in health care: reflections in the production of functioning and disability data. Fisioter Mov. 2020;33:19. doi: https://doi.org/10.1590/1980-5918.033.ao21 